\title{
Réflexions dans un cristal
}

\author{
Pierre Baumann, Stéphane Gaussent et Joel Kamnitzer
}

\begin{abstract}
Résumé
Soit $\mathfrak{g}=\mathfrak{n}^{-} \oplus \mathfrak{h} \oplus \mathfrak{n}^{+}$une algèbre de Kac-Moody symétrisable. Soit $B(\infty)$ le cristal de Kashiwara de $U_{q}\left(\mathfrak{n}^{-}\right)$, soit $\lambda$ un poids dominant, soit $T_{\lambda}=\left\{t_{\lambda}\right\}$ le cristal à un élément de poids $\lambda$, et soit $B(\lambda) \subseteq B(\infty) \otimes T_{\lambda}$ le cristal de la représentation intégrable de plus haut poids $\lambda$. Nous calculons les paramètres en cordes descendants d'un élément $b \otimes t_{\lambda}$ de $B(\lambda)$ en fonction des paramètres de Lusztig de $b$.
\end{abstract}

\section{1 Énoncé du résultat}

Soit $\mathfrak{g}$ une algèbre de Kac-Moody symétrisable. Elle vient avec la décomposition triangulaire $\mathfrak{g}=\mathfrak{n}^{-} \oplus \mathfrak{h} \oplus \mathfrak{n}^{+}$, la famille $\left(\alpha_{1}, \ldots, \alpha_{n}\right)$ des racines simples, la famille $\left(\alpha_{1}^{\vee}, \ldots, \alpha_{n}^{\vee}\right)$ des coracines simples, et le groupe de Weyl $W$ engendré par les réflexions simples $s_{1}, \ldots, s_{n}$. Soit $P^{+}$ l'ensemble des poids entiers dominants.

A chaque $\lambda \in P^{+}$correspond une représentation irréductible intégrable $L(\lambda)$ de $\mathfrak{g}$. Le choix d'un vecteur de plus haut poids $v_{\lambda}$ donne lieu à une surjection $X \mapsto X \cdot v_{\lambda}$ de $U\left(\mathfrak{n}^{-}\right)$sur $L(\lambda)$. La combinatoire de cette situation est régie par les cristaux de Kashiwara [6] : le cristal $B(\lambda)$ de $L(\lambda)$ est un sous-cristal de $B(\infty) \otimes T_{\lambda}$, où $B(\infty)$ est le cristal de $U_{q}\left(\mathfrak{n}^{-}\right)$et $T_{\lambda}=\left\{t_{\lambda}\right\}$ est le cristal à un élément de poids $\lambda$. Le cristal $B(\lambda)$ étant normal, nous avons

$$
\varphi_{i}\left(b \otimes t_{\lambda}\right)=\max \left\{n \in \mathbb{N} \mid \tilde{f}_{i}^{n}\left(b \otimes t_{\lambda}\right) \in B(\lambda)\right\}
$$

pour tout $i \in\{1, \ldots, n\}$ et tout $b \otimes t_{\lambda} \in B(\lambda)$.

Le cristal $B(\infty)$ est muni d'une involution * (voir [6], §8.3), ce qui permet de définir des opérateurs $\tilde{e}_{i}^{*}=* \tilde{e}_{i} *$. Dans [11], Saito définit une bijection

$$
\sigma_{i}:\left\{b \in B(\infty) \mid \varepsilon_{i}(b)=0\right\} \rightarrow\left\{b \in B(\infty) \mid \varepsilon_{i}\left(b^{*}\right)=0\right\}
$$

$\operatorname{par} \sigma_{i}(b)=\tilde{f}_{i}^{\varphi_{i}\left(b^{*}\right)} \tilde{e}_{i}^{* \max } b$. Prolongeons $\sigma_{i}$ à $B(\infty)$ en posant $\hat{\sigma}_{i}(b)=\sigma_{i}\left(\tilde{e}_{i}^{\max } b\right)$ pour tout $b \in B(\infty)$. 
Théorème 1 Soit $\lambda \in P^{+}$, soit $\left(s_{i_{1}}, \ldots, s_{i_{\ell}}\right)$ une décomposition réduite dans $W$, et soit $b \in$ $B(\infty)$ tel que $b \otimes t_{\lambda} \in B(\lambda)$. Définissons par récurrence des éléments $b_{0}, \ldots, b_{\ell}$ de $B(\infty)$ et des entiers $c_{1}, \ldots, c_{\ell}$ par

$$
b_{0}=b, \quad c_{k}=\varphi_{i_{k}}\left(b_{k-1} \otimes t_{\lambda}\right), \quad b_{k} \otimes t_{\lambda}=\tilde{f}_{i_{k}}^{c_{k}}\left(b_{k-1} \otimes t_{\lambda}\right),
$$

pour tout $k \in\{1, \ldots, \ell\}$. Posons enfin $d_{k}=\left\langle\alpha_{i_{k}}^{\vee}, s_{i_{k-1}} \cdots s_{i_{1}} \lambda\right\rangle$. Alors

$$
\hat{\sigma}_{i_{\ell}} \cdots \hat{\sigma}_{i_{1}} b=\tilde{e}_{i_{\ell}}^{* d_{\ell}} \cdots \tilde{e}_{i_{1}}^{* d_{1}} b_{\ell} .
$$

Le cas particulier $\ell=1$ de ce résultat est dû à Muthiah et Tingley ([10], proposition 2.2).

Plaçons-nous sous les hypothèses du théorème 1 . Prenant $k \in\{1, \ldots, \ell\}$, écrivant (1) pour la décomposition réduite $\left(s_{i_{1}}, \ldots, s_{i_{k}}\right)$, et égalant les poids des deux membres, nous trouvons

$$
s_{i_{1}} \cdots s_{i_{k}} \operatorname{wt}\left(\hat{\sigma}_{i_{k}} \cdots \hat{\sigma}_{i_{1}} b\right)+\lambda=s_{i_{1}} \cdots s_{i_{k}} \operatorname{wt}\left(b_{k} \otimes t_{\lambda}\right) .
$$

Notant $n_{1}, \ldots, n_{\ell}$ les entiers solutions du système d'équations

$$
s_{i_{1}} \cdots s_{i_{k}} \operatorname{wt}\left(\hat{\sigma}_{i_{k}} \cdots \hat{\sigma}_{i_{1}} b\right)-\operatorname{wt}(b)=\sum_{p=1}^{k} n_{p} s_{i_{1}} \cdots s_{i_{p-1}} \alpha_{i_{p}},
$$

pour $k \in\{1, \ldots, \ell\}$, nous obtenons alors

$$
n_{k}=-c_{k}-\left\langle\alpha_{i_{k}}^{\vee}, \mathrm{wt}\left(b_{k} \otimes t_{\lambda}\right)\right\rangle
$$

Supposons qu'en outre $\mathfrak{g}$ soit de dimension finie, et prenons pour $\left(s_{i_{1}}, \ldots, s_{i_{\ell}}\right)$ une décomposition réduite de l'élement le plus long de $W$. Il suit alors de [11] que les entiers $n_{1}, \ldots, n_{\ell}$ sont les paramètres de Lusztig de $b$ relativement à cette décomposition réduite (voir par exemple [2], §3 pour cette notion). Dans ces conditions, la formule (3) est équivalente à la relation découverte par Morier-Genoud [9] entre les paramètres de Lusztig de $b$ et les paramètres en cordes de $b^{\prime}$, où $b^{\prime} \otimes t_{\lambda}$ est l'image de $b \otimes t_{\lambda}$ par l'involution de Schützenberger de $B(\lambda)$.

Toujours dans le cas où $\mathfrak{g}$ est de dimension finie, l'égalité (2) reflète la possibilité d'exprimer les sommets du polytope de Mirković-Vilonen de $b \otimes t_{\lambda}$ de deux façons différentes : soit en termes de réflexions de Saito, autrement dit en termes de paramètres de Lusztig, soit en termes de la structure de cristal de $B(\lambda)$ (voir [4], §6). La principale motivation du présent travail réside dans la possibilité d'étendre partiellement ce résultat au cas où $\mathfrak{g}$ est une algèbre de Kac-Moody symétrisable. 


\section{Démonstration}

Ramenons-nous au cas d'une matrice de Cartan symétrique grâce à la méthode de repliement du diagramme de Dynkin (voir par exemple [7], §5). Soit $\Lambda$ la $\mathbb{C}$-algèbre préprojective complétée construite sur le graphe de Dynkin de $\mathfrak{g}$. En vecteur-dimension $\nu$, les structures de $\Lambda$-module sont les points d'une variété affine $\Lambda(\nu)$, appelée variété nilpotente de Lusztig. Les composantes irréductibles de ces variétés sont indexées par $B(\infty)$ (voir [8], théorème 5.3.1) : à un élément $b \in B(\infty)$ de poids $-\nu$ est associée une composante irréductible $\Lambda_{b}$ de $\Lambda(\nu)$. Cette bijection permet de lire les opérations de cristal de $B(\infty)$ en termes d'opérations algébriques sur les $\Lambda$-modules. Le théorème 1 se trouve alors être la traduction du théorème 4 ci-dessous.

Pour $i \in\{1, \ldots, n\}$, notons $S_{i}$ le $\Lambda$-module simple de vecteur-dimension $\alpha_{i}$ et $I_{i}$ l'annulateur de $S_{i}$. Nous définissons le $i$-socle $\operatorname{soc}_{i} M$ (respectivement, la $i$-tête hd ${ }_{i} M$ ) d'un $\Lambda$-module $M$ comme étant le plus grand sous-module (respectivement, quotient) de $M$ isomorphe à une somme directe de copies de $S_{i}$. Alors $\operatorname{soc}_{i} M \cong \operatorname{Hom}_{\Lambda}\left(\Lambda / I_{i}, M\right)$ et hd $i \cong\left(\Lambda / I_{i}\right) \otimes_{\Lambda} M$.

Si $\left(s_{i_{1}}, \ldots, s_{i_{\ell}}\right)$ est une décomposition réduite dans $W$, alors le $\Lambda$-bimodule $I_{i_{1}} \otimes_{\Lambda} \cdots \otimes_{\Lambda} I_{i_{\ell}}$ est isomorphe à l'idéal produit $I_{i_{1}} \cdots I_{i_{\ell}}$ (voir [3]). Ce dernier ne dépend que du produit $w=$ $s_{i_{1}} \cdots s_{i_{\ell}}$ et peut donc être désigné par la notation $I_{w}$. Comme $I_{w}$ est basculant, la théorie de Brenner-Butler fournit deux paires de torsion $\left(\mathscr{T}_{w}, \mathscr{F}_{w}\right)$ et $\left(\mathscr{T}^{w}, \mathscr{F}^{w}\right)$ dans la catégorie des $\Lambda$-modules de dimension finie, données par

$$
\begin{aligned}
\mathscr{T}_{w} & =\left\{T \mid I_{w} \otimes_{\Lambda} T=0\right\}, & \mathscr{F}_{w} & =\left\{T \mid \operatorname{Tor}_{1}^{\Lambda}\left(I_{w}, T\right)=0\right\}, \\
\mathscr{T}^{w} & =\left\{T \mid \operatorname{Ext}_{\Lambda}^{1}\left(I_{w}, T\right)=0\right\}, & \mathscr{F}^{w} & =\left\{T \mid \operatorname{Hom}_{\Lambda}\left(I_{w}, T\right)=0\right\} .
\end{aligned}
$$

Le sous-module de torsion d'un $\Lambda$-module $M$ de dimension finie relativement à $\left(\mathscr{T}_{w}, \mathscr{F}_{w}\right)$ (respectivement, $\left(\mathscr{T}^{w}, \mathscr{F}^{w}\right)$ ) est noté $M_{w}$ (respectivement, $M^{w}$ ).

Lemme 2 Soit $w \in W$, soit $i \in\{1, \ldots, n\}$, et soit $M$ un $\Lambda$-module de dimension finie. Supposons que $s_{i} w>w$ et que $\operatorname{Ext}_{\Lambda}^{1}\left(S_{i}, M\right)=0$. Alors $M^{s_{i} w} \cong I_{i} \otimes_{\Lambda} M^{w}$.

Preuve. Pour commencer, observons que $\operatorname{soc}_{i} M \in \mathscr{T}_{s_{i}}$ ([1], exemple 5.6 (i)), d'où $I_{i} \otimes_{\Lambda} \operatorname{soc}_{i} M=$ 0. Posons $N=\operatorname{Hom}_{\Lambda}\left(I_{i}, M\right)$. Appliquant deux fois le théorème 5.4 (i) de [1], nous obtenons

$$
M^{s_{i} w} \cong I_{s_{i} w} \otimes_{\Lambda} \operatorname{Hom}_{\Lambda}\left(I_{s_{i} w}, M\right) \cong I_{i} \otimes_{\Lambda} I_{w} \otimes_{\Lambda} \operatorname{Hom}_{\Lambda}\left(I_{w}, N\right) \cong I_{i} \otimes_{\Lambda} N^{w} .
$$

Compte tenu de l'isomorphisme $S_{i} \cong \Lambda / I_{i}$, l'hypothèse $\operatorname{Ext}_{\Lambda}^{1}\left(S_{i}, M\right)=0$ conduit à la suite exacte $0 \rightarrow \operatorname{soc}_{i} M \rightarrow M \rightarrow N \rightarrow 0$. L'hypothèse $s_{i} w>w$ entraîne que $\mathscr{T}_{i} \subseteq \mathscr{T}^{w}$ ([1], proposition 5.16), d'où $\operatorname{soc}_{i} M \in \mathscr{T}^{w}$. Nous avons ainsi une suite exacte $0 \rightarrow \operatorname{soc}_{i} M \rightarrow M^{w} \rightarrow$ $N^{w} \rightarrow 0$, d'où nous déduisons que $I_{i} \otimes_{\Lambda} M^{w} \cong I_{i} \otimes_{\Lambda} N^{w} \cong M^{s_{i} w}$. 
Pour un $\Lambda$-module $M$ et un mot $\left(i_{1}, \ldots, i_{\ell}\right)$, définissons les sous-modules $\operatorname{soc}_{\left(i_{1}, \ldots, i_{k}\right)} M$ de $M$ par récurrence sur $k \in\{0, \ldots, \ell\}$ de la façon suivante $([5], \S 2.4)$ :

$$
\operatorname{soc}_{()} M=0, \quad \operatorname{soc}_{\left(i_{1}, \ldots, i_{k}\right)} M / \operatorname{soc}_{\left(i_{1}, \ldots, i_{k-1}\right)} M=\operatorname{soc}_{i_{k}}\left(M / \operatorname{soc}_{\left(i_{1}, \ldots, i_{k-1}\right)} M\right) .
$$

Pour $i \in\{1, \ldots, n\}$, notons $\hat{I}_{i}$ l'enveloppe injective de $S_{i}$. Pour $\lambda \in P^{+}$, posons $\hat{I}_{\lambda}=\bigoplus_{i=1}^{n} \hat{I}_{i}^{\oplus \lambda_{i}}$, où $\lambda_{i}=\left\langle\alpha_{i}^{\vee}, \lambda\right\rangle$.

Lemme 3 Soit $\lambda \in P^{+}$, soit $w \in W$, et soit $\left(s_{i_{1}}, \ldots, s_{i_{\ell}}\right)$ une décomposition réduite de $w$. Alors $\operatorname{soc}_{\left(i_{1}, \ldots, i_{\ell}\right)} \hat{I}_{\lambda}$ est le plus grand sous-module de $\hat{I}_{\lambda}$ appartenant à $\mathscr{T}_{w}$ et son vecteur-dimension est égal à $\lambda-w^{-1} \lambda$.

Preuve. L'énoncé équivaut à dire que $\operatorname{soc}_{\left(i_{1}, \ldots, i_{\ell}\right)} \hat{I}_{\lambda} \in \mathscr{T}_{w}$ et que $\operatorname{Hom}_{\Lambda}\left(X, \hat{I}_{\lambda} / \operatorname{soc}_{\left(i_{1}, \ldots, i_{\ell}\right)} \hat{I}_{\lambda}\right)=$ 0 pour tout module $X \in \mathscr{T}_{w}$. Par additivité, nous pouvons donc nous ramener au cas où $\lambda$ est un poids fondamental, c'est-à-dire $\hat{I}_{\lambda}$ est un module indécomposable $\hat{I}_{j}$.

Le module $\operatorname{soc}_{\left(i_{1}, \ldots, i_{\ell}\right)} \hat{I}_{j}$ est le module noté $I_{\mathbf{i}, j}$ dans [5], $\$ 2.4$, avec $\mathbf{i}=\left(i_{1}, \ldots, i_{\ell}\right)$. C'est un objet injectif de la catégorie $\mathscr{T}_{w}$ ([5], théorème 2.8 (iii) et [1], exemple 5.15). Montrons qu'il contient tous les sous-modules de $\hat{I}_{j}$ appartenant à $\mathscr{T}_{w}$. Soit $X$ un tel sous-module. Alors la somme $Y=X+\operatorname{soc}_{\left(i_{1}, \ldots, i_{\ell}\right)} \hat{I}_{j}$ appartient à $\mathscr{T}_{w}$. Comme $\operatorname{soc}_{\left(i_{1}, \ldots, i_{\ell}\right)} \hat{I}_{j}$ est injectif dans $\mathscr{T}_{w}$, il est un facteur direct de $Y$. Or tous les sous-modules non-nuls de $\hat{I}_{j}$ contiennent son socle $S_{j}$, ce qui exclut l'existence de somme directe non-triviale à l'intérieur de $\hat{I}_{j}$. Par conséquent, $\operatorname{soc}_{\left(i_{1}, \ldots, i_{\ell}\right)} \hat{I}_{j}$ est soit égal à $Y$ tout entier, soit réduit à 0 . La seconde possibilité a lieu quand $j \notin\left\{i_{1}, \ldots, i_{\ell}\right\}$, mais dans ce cas $X$ est lui aussi nul, car pour des raisons de vecteur-dimension, il ne peut pas contenir le socle $S_{j}$ de $\hat{I}_{j}$ ([5], corollaire 9.3 et lemme 10.2). Donc $\operatorname{soc}_{\left(i_{1}, \ldots, i_{\ell}\right)} \hat{I}_{j}$ contient $X$ dans les deux cas de figure.

Enfin, l'assertion sur le vecteur-dimension est prouvée dans [5], corollaire 9.2.

Théorème 4 Soit $\lambda \in P^{+}$, soit $w \in W$, soit $\left(s_{i_{1}}, \ldots, s_{i_{\ell}}\right)$ une décomposition réduite de $w$, et soit $M$ un sous-module de dimension finie de $\hat{I}_{\lambda}$. Construisons par récurrence une chaîne $M_{0} \subseteq M_{1} \subseteq \cdots \subseteq M_{\ell}$ de sous-modules de $\hat{I}_{\lambda}$ de la façon suivante :

$$
M_{0}=M, \quad M_{k} / M_{k-1}=\operatorname{soc}_{i_{k}}\left(\hat{I}_{\lambda} / M_{k-1}\right) .
$$

Alors $\left(M_{\ell}\right)_{w}=\operatorname{soc}_{\left(i_{1}, \ldots, i_{\ell}\right)} \hat{I}_{\lambda}$ et $M_{\ell} /\left(M_{\ell}\right)_{w} \cong \operatorname{Hom}_{\Lambda}\left(I_{w}, M\right)$.

Preuve. Des arguments classiques montrent que si $f: X \rightarrow Y$ est un homomorphisme de $\Lambda$-modules, alors $f\left(\operatorname{soc}_{\left(i_{1}, \ldots, i_{\ell}\right)} X\right) \subseteq \operatorname{soc}_{\left(i_{1}, \ldots, i_{\ell}\right)} Y$. Appliquant ce résultat à la surjection $\hat{I}_{\lambda} \rightarrow$ 
$\hat{I}_{\lambda} / M$, nous obtenons que $\operatorname{soc}_{\left(i_{1}, \ldots, i_{\ell}\right)} \hat{I}_{\lambda}$ est inclus dans $M_{\ell}$. La première égalité de l'énoncé découle alors du lemme 3 .

Par ailleurs, $S_{i_{k}} \in \mathscr{F}^{s_{i_{k}} \cdots s_{i_{\ell}}}$ pour tout $k \in\{1, \ldots, \ell\}$, d'où $\left(M_{k-1}\right)^{s_{i_{k}} \cdots s_{i_{\ell}}}=\left(M_{k}\right)^{s_{i_{k}} \cdots s_{i_{\ell}}}$. La définition de $M_{k}$ entraîne que $\operatorname{soc}_{i_{k}}\left(\hat{I}_{\lambda} / M_{k}\right)=0$, autrement dit $\operatorname{Hom}_{\Lambda}\left(S_{i_{k}}, \hat{I}_{\lambda} / M_{k}\right)=0$; compte tenu du caractère injectif de $\hat{I}_{\lambda}$, cela donne $\operatorname{Ext}_{\Lambda}^{1}\left(S_{i_{k}}, M_{k}\right)=0$. Une utilisation répétée du lemme 2 conduit alors à $M^{w} \cong I_{w} \otimes_{\Lambda} M_{\ell}$. Utilisant les relations $M_{\ell} /\left(M_{\ell}\right)_{w} \cong \operatorname{Hom}_{\Lambda}\left(I_{w}, I_{w} \otimes_{\Lambda}\right.$ $\left.M_{\ell}\right)$ ([1], théorème 5.4 (ii)) et $\operatorname{Hom}_{\Lambda}\left(I_{w}, M / M^{w}\right)=0$, nous obtenons la seconde égalité de l'énoncé.

Nous pouvons maintenant démontrer le théorème 1. Adoptons les notations utilisées dans son énoncé. Soit $M$ un point général de la composante irréductible $\Lambda_{b}$. La condition $b \otimes$ $t_{\lambda} \in B(\lambda)$ se traduit par $\varepsilon_{i}\left(b^{*}\right) \leq\left\langle\alpha_{i}^{\vee}, \lambda\right\rangle$ pour tout $i \in\{1, \ldots, n\}$ ([6], proposition 8.2), d'où $\operatorname{dim} \operatorname{soc}_{i} M \leq \operatorname{dim} \operatorname{soc}_{i} \hat{I}_{\lambda}$. Il existe donc une inclusion $M \hookrightarrow \hat{I}_{\lambda}$. Les modules $M_{k}$ construits dans l'énoncé du théorème 4 sont alors des points généraux des composantes $\Lambda_{b_{k}}$ et le module $\operatorname{Hom}_{\Lambda}\left(I_{w}, M\right)$ est un point général de $\Lambda_{\hat{\sigma}_{i_{\ell}} \cdots \hat{\sigma}_{i_{1}} b}$ ([1], proposition 5.24). Il reste à observer que $\operatorname{soc}_{\left(i_{1}, \ldots, i_{k}\right)} \hat{I}_{\lambda} / \operatorname{soc}_{\left(i_{1}, \ldots, i_{k-1}\right)} \hat{I}_{\lambda}$ est isomorphe à la somme directe de $d_{k}$ copies de $S_{i_{k}}$.

\section{Références}

[1] P. Baumann, J. Kamnitzer, P. Tingley, Affine Mirković-Vilonen polytopes, prépublication arXiv:1110.3661.

[2] A. Berenstein and A. Zelevinsky, Tensor product multiplicities, canonical bases and totally positive varieties, Invent. Math. 143 (2001), 77-128.

[3] A. B. Buan, O. Iyama, I. Reiten, J. Scott, Cluster structures for 2-Calabi-Yau categories and unipotent groups, Compos. Math. 145 (2009), 1035-1079.

[4] M. Ehrig, MV-polytopes via affine buildings, Duke Math. J. 155 (2010), 433-482.

[5] C. Geiß, B. Leclerc, J. Schröer, Kac-Moody groups and cluster algebras, Adv. Math. 228 (2011), 329-433.

[6] M. Kashiwara, On crystal bases, in: Representations of groups (Banff, 1994), CMS Conf. Proc. vol. 16, American Mathematical Society, 1995, pp. 155-197.

[7] M. Kashiwara, Similarity of crystal bases, in: Lie algebras and their representations (Seoul, 1995), Contemp. Math. vol. 194, American Mathematical Society, 1996, pp. 177-186.

[8] M. Kashiwara, Y. Saito, Geometric construction of crystal bases, Duke Math. J. 89 (1997), 9-36.

[9] S. Morier-Genoud, Relèvement géométrique de la base canonique et involution de Schützenberger, C. R. Acad. Sci. Paris, Ser. I 337 (2003), 371-374. 
[10] D. Muthiah, P. Tingley, Affine PBW Bases and MV Polytopes in Rank 2, prépublication arXiv:1209.2205.

[11] Y. Saito, $P B W$ basis of quantized universal enveloping algebras, Publ. Res. Inst. Math. Sci. 30 (1994), 209-232.

Pierre Baumann, Institut de Recherche Mathématique Avancée, Université de Strasbourg et CNRS, 7 rue René Descartes, 67084 Strasbourg Cedex, France.

p.baumann@unistra.fr

Stéphane Gaussent, Université de Lyon, ICJ (UMR 5208), Université Jean Monnet, 42023 Saint-Etienne Cedex 2, France.

stephane.gaussent@univ-st-etienne.fr

Joel Kamnitzer, Department of Mathematics, University of Toronto, Toronto, ON, M5S 2E4 Canada.

jkamnitz@math.toronto.edu 